\title{
Evaluation of oxidative and nitrosative stress in relapsing remitting multiple sclerosis: effect of corticosteroid therapy
}

\author{
Arzu Seven', Mahmure Aslan², Said İncir3 , Ayşe Altıntaş4 \\ 1Department of Biochemistry, Medical Faculty, İstanbul University, ${ }^{2}$ Hospitalium Hospital, Șișli, İstanbul, ${ }^{3}$ Biochemistry Department, \\ Cerrahpașa Medical Faculty, İstanbul University, ${ }^{4}$ Neurology Department, Cerrahpașa Medical Faculty, istanbul University, Turkey
}

\begin{abstract}
This study is designed to evaluate the roles of oxidative and nitrosative stress in serum and cerebrospinal fluid (CSF) of relapsing remitting multiple sclerosis (RRMS) patients. Oxidative stress markers thiobarbituric acid reactive substances (TBARS), 8-epi-PGF2 $\alpha$, conjugated diene and nitrosative stress markers nitrotyrosine, nitrit-nitrate were analysed in serum and CSF of 20 newly diagnosed RRMS patients before and after methyl prednysolone (MP) therapy (1000 mg/day i.v., for 5 days) and in healthy control group.TBARS and conjugated diene were analysed spectrophotometrically, nitritenitrate fluorometrically, 8-epi-PGF2 $\alpha$ and nitrotyrosine were measured by ELISA.

Serum conjugated diene $(p<0.001)$ and 8 -epi-PGF2 $\alpha(p<0.05)$ levels were significantly higher in RRMS patients before MP therapy with respect to control group. MP therapy caused a significant decrease only in 8-epi-PGF2a level $(p<0.05)$. Serum nitrotyrosine levels were significantly lower in RRMS patients both before $(p<0.001)$ and after $(p<0.001)$ MP therapy with respect to controls. Serum nitrite-nitrate levels were significantly lower $(p<0.05)$ in RRMS patients before therapy compared to controls. Nitrotyrosine and nitrite-nitrate levels in CSF of RRMS patients were significantly higher $(p<0.001)$ before therapy compared to normal pressure hydrocephalia control group.

Our findings reveal increased oxidative stress in serum of RRMS patients and the benefical role of MP therapy in relieving oxidative stress.As to nitrosative stress, nitrotyrosine and nitrite-nitrate levels were increased in CSF and decreased in serum.
\end{abstract}

Key words: multiple sclerosis, oxidative/nitrosative stress, CSF, methylprednisolone.

\section{Introduction}

Multiple sclerosis (MS) is a chronic complex neurological disease with a variable clinical course and several pathophysiological mechanisms such as inflammation, demyelination, axonal/neuronal damage, gliosis, remyelination and repair mechanisms, oxidative injury and excitotoxicity. Alterations of the immune system together with biochemical disturbances and disruption of the blood-brain barrier are also involved in the pathomechanisms [27]. These processes are not uniformly represented in patient populations but can selectively predominate in individual patients. Therefore, heterogeneity in phenotypic expression of MS has potential effects on the prognosis and response to therapies in MS patients. Relapsing remitting mul- 
tiple sclerosis (RRMS) is characterised by periods of clinical stability, punctuated by subacute attacks of clinical worsening after complete or partial recovery [28].

Oxidative stress, which is usually defined as a preponderance of the production of free radicals over their elimination, is commonly implicated in the development of brain damage. Active inflammatory processes predominating in the relapse phase of RRMS lead to the generation of highly toxic reactive oxygen species (ROS) and reactive nitrogen species (RNS) and release of a great number of inflammatory mediators and cytokines. Polyunsaturated fatty acids (PUFA), main components of neuron membranes, are highly susceptible to ROS attack. Free radical catalysed peroxidation of arachidonic acid esterified in membrane phospholipids leads to the formation of isoprostanes, which are emerging as prostaglandin-like compounds. Isoprostanes are sensitive and specific markers of in vivo lipid peroxidation and oxidative tissue injury [22].

In RRMS, activated glia secrete RNS, which can cause nitrosative stress, resulting in myelin destruction. Due to the elevated expression of NOS in activated microglia and infiltrated macrophages, nitric oxide (NO) production is significantly increased in the inflammatory region. Evidence suggests that NO contributes to tissue injury, blood-brain barrier (BBB) breakdown, axonal degeneration and plaque formation. On the other hand, NO may help to control the immune response via several immunoregulatory processes. Nitration of tyrosine residues by NO-derived species results in the accumulation of 3-nitrotyrosine in proteins. It is a marker of nitrosative stress in cells and tissues and also a relatively specific marker of oxidative damage, mediated by peroxynitrite, which is formed by the reaction of superoxide with NO $[6,7]$.

The central nervous system (CNS) is particularly susceptible to oxidative stress due to the high rate of oxygen utilization, a relatively poor antioxidant defence system and a high content of PUFA. Accumulation of iron in the brain, as a function of age, may also be a potent catalyst for oxidative species formation [15].

In the recent years, the role of glucocorticoids (GC) in the RRMS treatment has gained strength. Due to their strong anti-inflammatory action, they are used most effectively to overcome and shorten the duration of neurological symptoms during relapses. GC activity consists mainly of the induction of specific inflammation suppressing genes and repression of genes coding proteins involved in immune and inflammatory reactions. GC therapy results in inhibition of inflam- matory reactions, sealing of $\mathrm{BBB}$ and decreasing the magnetic resonance imaging (MRI) number of gadolinium up-taking foci [19].

This study is undertaken to elucidate further the roles of nitrosative and oxidative stress in RRMS patients and to investigate the beneficial role of methylprednisolone (MP) therapy. To serve these purposes, oxidative stress markers - thiobarbituric acid reactive substances (TBARS), conjugated diene and 8-epi-PG F2 $\alpha$ and nitrosative stress markers - nitrate-nitrite and nitrotyrosine - were analysed both in the serum and cerebrospinal fluid (CFS).

\section{Material and methods \\ Patients}

Twenty clinically definite RRMS patients, newly diagnosed according to Mc Donald diagnostic criteria [16], were enrolled in this study. The relapsing remitting course of multiple sclerosis patients was ascertained according to Lubin et al. [14]. The clinical diagnosis of RRMS patients, admitted to the Neurology Department of Marmara University, was based on clinical signs, routine diagnostic CSF and blood tests, full neurological examination and MRI of the central nervous system. The neurologic deficits were scored with the Kurtzke expanded disability status scale (EDSS) [13]. RRMS patients with symptoms persisting for more than 24 hours were considered as having acute attack and were subject to a high dose of MP (1000 mg/day) i.v. infusions for 5 consecutive days. Relapse was defined as worsening of EDSS by 1.0 point, new clinical symptoms of subjective character or objectively present, lasting at least 24 hours, in the absence of infection or fever, after a period of at least 30 days of neurological stability.

RRMS group consisted of 13 females and 7 males. Mean age was $31.00(S D=9.6)$ years, mean EDSS score was 1.70 (SD = 0.84). There were two control groups. The first control group consisted of 10 females and 5 males. Mean age was $30.20 \pm 5.51$. Individuals with normal pressure hydrocephalus (9 females, 6 males; mean age: $28.33 \pm 5.31$ ) formed the second control group for CSF analyses.

Patients with kidney, liver, endocrine, immunological, inflammatory or infectious disorders were excluded on the basis of history, physical examinations and laboratory evaluations. Patients were not on anti-inflammatory, immunosuppressive, immunomodulatory, vitamin, antioxidant, steroid or hormonal treatment for at least three months before their inclusion in the study. 
All healthy controls were screened to be free from any neurological or other major medical illnesses. The study protocol was approved by the Ethics Committee of Marmara Faculty. Written informed consent was obtained from all patients and controls.

\section{Biochemical investigation}

Blood samples, with and without EDTA, were collected between 8 and 9 AM after an overnight fast, within 7 days of an acute exacerbation and 3 days after MP therapy, twice from RRMS patients and once from controls. Serum and plasma samples were stored at $-70^{\circ} \mathrm{C}$ until analyses. CSF samples were taken within 7 days of an acute exacerbation, before MP therapy from RRMS patients and from individuals with normal pressure hydrocephalus, forming the control group. CSF samples were collected by lumbar puncture in the $L 3 / L 4$ or L4/L5 interspace. The first $12 \mathrm{ml}$ of CSF was collected in a polypropylene tube, immediately centrifuged at $2000 \times \mathrm{g}$, at $+4^{\circ} \mathrm{C}$ for $10 \mathrm{~min}$. The supernatant was pipetted off, gently mixed to avoid possible gradient effects and aliquoted in polypropylene tubes that were stored at $-80^{\circ} \mathrm{C}$, pending biochemical analyses, without being thawed and refrozen.

\section{Oxidative stress parameters}

1. TBARS was determined spectrophotometrically by the method of Buege and Aust [2]. One volume of plasma was mixed thoroughly with 2 vol. stock solution of $15 \% \mathrm{w} / \mathrm{v}$ trichloroacetic acid, $0.375 \% \mathrm{w} / \mathrm{v}$ thiobarbituric acid and $0.25 \mathrm{~N}$ hydrochloric acid. The mixture was heated for 30 min in a boiling water bath. After cooling, the flocculent precipitate was removed by centrifugation at $1000 \times \mathrm{g}$ for $10 \mathrm{~min}$. The light $\mathrm{ab}$ sorbance of the sample was determined at $535 \mathrm{~nm}$. and TBARS concentration was calculated using $1.56 \times 105 \mathrm{M}^{-1} \mathrm{~cm}^{-1}$ as molar extinction coefficient.

2. Conjugated diene was determined according to the method of Slater [25]. Lipids were extracted with chloroform-methanol (2:1), followed by centrifugation at $1000 \times \mathrm{g}$ for $5 \mathrm{~min}$. The chloroform layer was evaporated to dryness under a stream of nitrogen. The lipid residue was dissolved in $1.5 \mathrm{~mL}$ cyclohexane and the absorbance read at $233 \mathrm{~nm}$ measured the amount of hydroperoxide formed.

3. 8-epi-PGF2 $\alpha$ levels were measured by a competitive enzyme-linked immunoassay (ELISA) (Cell Biolabs Inc. Oxiselect). The antibody to 8-isoprostane levels is incubated in precoated microtitre plate wells. Upon washing, 8-epi-PGF2 $\alpha$ standards or treated samples were mixed with 8-epi-PGF2 $\alpha$ conjugated to horseradish peroxidase (HRP) and added simultaneously to the wells. Free 8-isoprostane and 8-epi-PGF2 $\alpha$ HRP conjugate compete to bind to the antibody bound on the plate.

8-epi-PGF2 $\alpha$ content in an unknown sample is determined by comparing with the known predetermined standard curve (detection limit: $2.7 \mu \mathrm{g} / \mathrm{ml}$, specificity with respect to other $P G s$ is $0.02 \%$ and $0.16 \%$ for PGE2 and PGD2, respectively).

\section{Nitrosative stress parameters}

1. Plasma and CSF 3-nitrotyrosine levels were measured by solid-phase enzyme-linked immunosorbent assay (ELISA), based on the sandwich principle, using streptavidin-peroxidase conjugate that binds to biotinylated tracer antibody and reacts with the substrate tetramethylbenzidine (TMB) (nitrotyrosine ELISA kit, HK 501, Hycult Biotechnology).

2. Total oxidative products of nitric oxide (NO) - nitrate plus nitrite - were measured fluorometrically after reduction of nitrate to nitrite by nitrate reductase. Total nitrites were converted in the diazonium salt by the addition of sulphanilamide, and then into azo dye derivate by adding $N$-(I-naphthyl)-ethylene-diamine.

\section{Data analysis}

The results were expressed as means \pm SD. The statistical significance of the differences was determined by analysis of variance (ANOVA), followed by student's $t$ test for parametric data. A value of $p<0.05$ was regarded as significant. Pearson's rank correlation was used for correlation analyses.

\section{Results}

Among oxidative stress parameters, serum TBARS values did not differ significantly between the study groups. Serum conjugated diene levels were significantly higher in RRMS patients both before therapy ( $p<0.001)$ and after therapy $(p<0.01)$ as compared to controls. MP therapy did not cause a significant decrease in the conjugated diene levels in RRMS patients. Serum 8-epi-PGF2 $\alpha$ levels were observed to be significantly higher $(p<0.05)$ in RRMS patients before therapy, compared to healthy controls. RRMS patients were observed to have significantly decreased 8-epi- 
Table I. Values of blood oxidative and nitrosative stress markers and statistical significance in RRMS patients before and after MP therapy and in control group

\begin{tabular}{|lccc|}
\hline & Control group $(n=15)$ & \multicolumn{2}{c|}{ RRMS group $(n=20)$} \\
\cline { 3 - 4 } & & before therapy & after therapy \\
\hline TBARS $(\mathrm{nmol} / \mathrm{ml})$ & $4.49 \pm 1.34$ & $5.23 \pm 1.67$ & $4.83 \pm 1.51$ \\
\hline 8 -epi-PGF2 $\alpha(\mathrm{ng} / \mathrm{ml})$ & $177.90 \pm 55.48^{\mathrm{a}^{*}}$ & $229.02 \pm 66.69$ & $186.34 \pm 35.87^{* *}$ \\
\hline Conjugated diene $(\mu \mathrm{mol} / \mathrm{L})$ & $129.48 \pm 78.54^{\mathrm{a}^{* * *}, \mathrm{b**}}$ & $226.98 \pm 78.20$ & $222.30 \pm 95.27$ \\
\hline Nitrotyrosine $(\mathrm{nM})$ & $933.59 \pm 233.33^{* * * *, \mathrm{~b} * * *}$ & $689.79 \pm 276.39$ & $598.64 \pm 270.53$ \\
\hline Nitrite-nitrate $(\mu \mathrm{mol} / \mathrm{L})$ & $76.61 \pm 21.68^{\mathrm{a}^{*}}$ & $63.07 \pm 11.79$ & $61.69 \pm 25.18$ \\
\hline
\end{tabular}

$a$ - Control vs RRMS patients before MP therapy, ${ }^{b}$ - Control vs RRMS patients after MP therapy, ${ }^{c}-$ RRMS patients before and after therapy ${ }^{*} p<0.05,{ }^{* *} p<0.01,{ }^{* * *} p<0.001$

PGF2 $\alpha$ levels $(p<0.05)$ after MP therapy compared to levels before therapy (Table I).

As to nitrosative stress parameters; serum nitrotyrosine levels were significantly lower in RRMS patients both before $(p<0.001)$ and after $(p<0.001)$ MP therapy as compared to control group. However, there was no significant change in nitrotyrosine values before and after therapy. Nitrite-nitrate levels were significantly lower $(p<0.05)$ in RRMS patients before therapy as compared to controls (Table I).

There was no significant difference in oxidative stress parameters analyzed in CSF among study groups. However, nitrosative stress markers; CSF nitrotyrosine, and CSF nitrite-nitrate levels were observed to be significantly higher $(p<0.001)$ in CSF of RRMS patients before therapy compared to normal pressure hydrocephalus control group (Table II).

Correlation analysis revealed positive correlations between age and conjugated diene $(r=0.446, p<0.05)$ and negative correlation between 8 -epi-PGF2 $\alpha$ and nitrotyrosine $(r=-0.510, p<0.05)$ in RRMS patients before MP therapy. Conjugated diene and TBARS were found to be positively correlated $(r=0.45, p<0.05)$ in RRMS patients after MP therapy.

\section{Discussion}

More than $80 \%$ of MS patients have a RRMS disease course with defined clinical exacerbations of neurologic symptoms, followed by complete/incomplete remissions. Oxidative and nitrosative stress can be placed as unifying processes in the centre of pathogenic mechanisms that lead to axonal/neuronal loss/damage and neurodegeneration in RRMS $[26,27]$.
Oxidative stress is believed to contribute to tissue injury in focal inflammatory lesions and to be involved in diffuse axonal degeneration. The determination of lipid peroxidation products relies on various methods. The most widely used marker of lipid peroxidation is the level of malondialdehyde (MDA) or conjugated dienes in plasma or red blood cells. The commonly used assay for MDA is based on the reaction with thiobarbituric acid and formation of TBARS. However, this method is not sensitive and is non-specific due to other metabolites that can react with thiobarbituric acid $[1,10]$.

Supporting the above information, the findings of our study showed that lipid peroxidation estimated by TBARS generation in RRMS patients are not significantly different from the healthy control group.

Table II. Values of CSF oxidative and nitrosative stress markers and statistical significance in RRMS patients and normal pressure hydrocephalia control group

\begin{tabular}{|lcc|}
\hline & $\begin{array}{c}\text { Normal pressure } \\
\text { hydrocephalia } \\
\text { control group } \\
(n=15)\end{array}$ & $\begin{array}{c}\text { RRMS patients } \\
\text { before MP } \\
\text { therapy } \\
(n=20)\end{array}$ \\
\hline TBARS $(\mathrm{nmol} / \mathrm{ml})$ & $0.59 \pm 0.24$ & $0.45 \pm 0.23$ \\
\hline 8-epi-PGF2 $\alpha(\mathrm{ng} / \mathrm{ml})$ & $141.26 \pm 42.83$ & $149.93 \pm 45.39$ \\
\hline $\begin{array}{l}\text { Conjugated diene } \\
(\mu \mathrm{mol} / \mathrm{L})\end{array}$ & $84.24 \pm 52.17$ & $122.85 \pm 104.61$ \\
\hline $\begin{array}{l}\text { Nitrotyrosine }(\mathrm{nM}) \\
573.54 \pm 142.86^{* * *}\end{array}$ & $927.89 \pm 244.84$ \\
\hline $\begin{array}{l}\text { Nitrite-nitrate } \\
(\mu m o l / L)\end{array}$ & $52.52 \pm 16.50^{* * *}$ & $86.28 \pm 34.10$ \\
\hline$* * * p<0.001$ & & \\
\hline
\end{tabular}


Because of its stability, F2-isoprostane measurement is widely considered to be the gold standard index of lipid peroxidation in vivo, as a sensitive index of degenerative phenomena, persisting in the absence of inflammatory activity. Our findings related to oxidative stress reveal significantly high serum 8-epi-PGF2 $\alpha$ and conjugated diene levels in RRMS patients before MP therapy as compared to controls. Elevated levels of 8 -epi-PGF2 $\alpha$ reflect free radical induced lipid peroxidation and oxidative stress. The source of isoprostanes may be oxidized arachidonic acid, derived not only from neuron membranes but also from membranes of different cells including erythrocytes. Furthermore, significantly reduced serum 8-epi-PGF2 $\alpha$ levels in response to MP therapy indicate both the sensitivity and reliability of isoprostane measurement and the antioxidant protective effects of steroid treatment on membrane phospholipids in addition to their anti-inflammatory activity $[4,8,17]$.

CSF isoprostane levels are widely considered as a reliable index of intracerebral oxidative injury [15,31]. In this study oxidative stress markers analysed in CSF of RRMS patients showed no significant differences with respect to control group. MS pathophysiology is likely to differ in different disease stages, thus the importance of oxidative stress in CSF could shift with disease subtypes.

In the literature, controversial findings are reported in relation to oxidative stress parameters analysed in serum and CSF of MS patients. Hunter et al. [9] reported significantly higher measures of lipid peroxidation in CSF but not in plasma of MS patients compared with the control group. Naidoo and Knapp [20] found significantly higher levels of TBARS in serum but not in CSF of MS patients, confirming the results of a study by Rogovino [25]. In the study of Keles et al. [11], MDA was reported to be significantly increased in both serum and CSF of MS patients before MP therapy compared to the control group and significantly decreased after MP therapy. Mitosek-Szewczyk et al. [19] observed significantly higher CSF and serum TBARS levels during relapse compared with controls. Their findings were in accordance with those of Koch et al. [12] and Ortiz et al. [23]. TBARS level in serum was reported to decrease after MP treatment and had the lowest value during the remission phase of MS compared with controls. In contrast to our findings, high isoprostane levels observed in CSF of RRMS patients were observed to be decreased after steroid treatment [8].
Nitric oxide has three major roles in biological systems, it can be protective with its antioxidant properties, it can be regulatory by altering vascular tone with a role in cell signalling, it can also be deleterious, causing DNA damage, lipid peroxidation and protein nitration. Due to lack of correlation between disease activity and NO, a dual role of $\mathrm{NO}$ as both immunoregulatory and proinflammatory is suggested in the pathogenesis of MS. Excess NO may be cytotoxic either by combining with tyrosine or by reacting with superoxide to form peroxynitrite. Increased production of NO for a prolonged time may contribute to oxidative-nitrative damage to cellular macromolecules including proteins. Nitration of tyrosine residues by NO-derived species results in the accumulation of 3-nitrotyrosine (3 NT) in proteins, as a footprint for peroxynitrite-mediated change in the cell and as an early marker of nitrosative stress and neurodegeneration. 3 NT formation is considered to be a posttranslational modification that may be responsible for lipid peroxidation and oxidative changes in plasma proteins $[3,21,24]$. Acute relapses of MS are characterised by complex immunological mechanisms involving blood-brain barrier damage and influx of peripheral blood cells. This causes focal inflammation and subsequent activation of resident glial cells, accompanied by the production and release of cytokines and other mediators. Activation of proinflammatory cytokines, such as TNF- $\alpha$ and INF- $\gamma$, induce iNOS potently and lead to increased CSF levels of NO, as a mediator of the inflammatory process [24].

In the presented study, nitrosative stress markers -3-NT and nitrite-nitrate - were found to be significantly lower in serum and higher in CSF of RRMS patients before MP therapy as compared to their control groups. MP therapy did not cause a significant change in nitrosative stress markers both in serum and CSF.

In the literature, contradictory findings related to nitrosative stress markers may be due to different analysis techniques for NO, nutrition of the patients, endothelium NO source, effects of gastrointestinal bacteria and pharmacotherapy [30]. As the blood arginine level and nitrogen in the diet may affect $\mathrm{NO}_{2}{ }^{-} / \mathrm{NO}_{3}{ }^{-}$ levels, CSF analysis of NO is reported to be a more reliable index [29]. Studies reporting increased CSF $\mathrm{NOx}$ (nitrite + nitrate) levels and decreased serum $\mathrm{NO}_{2}{ }^{-} / \mathrm{NO}_{3}{ }^{-}$levels [5] support our findings. However, contrary to our data; plasma and CSF nitrite and nitrate levels were observed to be elevated in MS in the studies conducted by C Larlori et al. [10] and Miller et al. [18]. 
Strengths of our study compared with previous studies are the selection of only RRMS patients as subtypes of MS and a higher number of patients. Using two different control groups for blood and CSF experiments may be regarded as a weakness for some researchers but we regard it as an ethic approach.

In diseases with complex pathogenesis like MS, an individual biomarker can reflect only one of many ongoing pathogenesis. Our findings related to blood and CSF oxidative stress markers suggest that lipid peroxidation is more prevalent in the periphery than in the CNS. In this respect, the level of isoprostanes may be used as a non-invasive marker to determine oxidative stress in RRMS patients. However, nitrosative stress markers seem to be increased in CSF and decreased in serum.

There appears to be no relation between oxidative/ nitrosative stress markers and disease course and progression of disability. Further research is needed to determine whether oxidative stress is an important feature of MS pathophysiology or more an epiphenomenon of neurodegeneration or inflammation.

\section{References}

1. Adibhatla RM, Hatcher JF. Phospholipase A(2), reactive oxygen species, and lipid peroxidation in CNS pathologies. BMB Rep 2008; 41: 560-567.

2. Beuge JA, Aust SD. Microsomal lipid peroxidation. Methods Enzymol 1978; 52: 302-310.

3. Bolaños JP, Heales SJ. Persistent mitochondrial damage by nitric oxide and its derivatives: neuropathological implications. Front Neuroenergetics 2010; 2: 1-5.

4. Cracowski JL, Durand T, Bessard G. Isoprostanes as a biomarker of lipid peroxidation in humans: physiology, pharmacology and clinical implications. Trends Pharmacol Sci 2002; 23: 360-366.

5. De Bustos F, Navarro JA, De Andrés C. Cerebrospinal fluid nitrate levels in patients with multiple sclerosis. Eur Neurol 1999; 41: 44-47.

6. Frischer JM, Bramow S, Dal-Bianco A, Lucchinetti CF, Rauschka H, Schmidbauer M, Laursen H, Sorensen PS, Lassmann H. The relation between inflammation and neurodegeneration in multiple sclerosis brains. Brain 2009; 132: 1175-1189.

7. Giovannoni G, Miller DH, Losseff NA, Sailer M, Lewellyn-Smith N, Thompson AJ, Thompson EJ. Serum inflammatory markers and clinical/MRI markers of disease progression in multiple sclerosis. J Neurol 2001; 248: 487-495.

8. Greco A, Minghetti L, Puopolo M, Cannoni S, Romano S, Pozzilli C, Levi G. Cerebrospinal fluid isoprostanes are not related to inflammatory activity in relapsing-remitting multiple sclerosis. J Neurol Sci 2004; 224: 23-27.

9. Hunter MIS, Nlemadim BC, Davidson DLW. Lipid peroxidation products and antioxidant proteins in plasma and cerebrospinal fluid from multiple sclerosis patients. Neurochem Res 1985; 10 : 1645-1652.
10. Iarlori C, Gambi D, Lugaresi A, Patruno A, Felaco M, Salvatore M, Speranza L, Reale M. Reduction of free radicals in multiple sclerosis: effect of glatiramer acetate (Copaxone). Mult Scler 2008; 14: 739-748.

11. Keles MS, Taysi S, Sen N, Aksoy H, Akçay F. Effect of corticosteroid therapy on serum and CSF malondialdehyde and antioxidant proteins in multiple sclerosis. Can J Neurol Sci 2001; 28: 141-143.

12. Koch M, Mostert J, Arutjunyan AV, Stepanov M, Teelken A, Heersema D, De Keyser J. Plasma lipid peroxidation and progression of disability in multiple sclerosis. Eur J Neurol 2007; 14: 529-533.

13. Kurtzke JF. Rating neurologic impairment in multiple sclerosis: an expanded disability status scale (EDSS). Neurology 1983; 33: 1444-1452.

14. Lublin FD, Reingold SC. Defining the clinical course of multiple sclerosis: results of an international survey. National Multiple Sclerosis Society (USA) Advisory Committee on Clinical Trials of New Agents in Multiple Sclerosis. Neurology 1996; 46: 907-911.

15. Mattsson N, Haghighi S, Andersen O, Yao Y, Rosengren L, Blennow K, Praticò D, Zetterberg H. Elevated cerebrospinal fluid F2-isoprostane levels indicating oxidative stress in healthy siblings of multiple sclerosis patients. Neurosci Lett 2007; 414: 233-236.

16. McDonald WI, Compston A, Edan G, Goodkin D, Hartung HP, Lublin FD, McFarland HF, Paty DW, Polman ChH, Reingold SC, Sandberg-Wollheim M, Sibley W, Thompson A, Van Den Noort S, Weinshenker BY, Wolinsky JS. Recommended diagnostic criteria for multiple sclerosis: Guidelines from the international panel on the diagnosis of multiple sclerosis. Ann Neurol 2001; 50: 121-127.

17. Miller E, Mrowicka M, Saluk-Juszczak J, Ireneusz M. The level of isoprostanes as a non-invasive marker for in vivo lipid peroxidation in secondary progressive multiple sclerosis. Neurochem Res 2011; 36: 1012-1016.

18. Miller E, Walczak A, Saluk J, Ponczek MB, Majsterek I. Oxidative modification of patient's plasma proteins and its role in pathogenesis of multiple sclerosis. Clin Biochem 2012; 45: 26-30.

19. Mitosek-Szewczyk K, Gordon-Krajcer W, Walendzik P, Stelmasiak Z. Free radical peroxidation products in cerebrospinal fluid and serum of patients with multiple sclerosis after glucocorticoid therapy. Folia Neuropathol 2010; 48: 116-122.

20. Naidoo R, Knapp ML. Studies of lipid peroxidation products in the cerebrospinal fluid and serum in multiple sclerosis and other conditions. Clin Chem 1992; 38: 2449-2454.

21. Nazliel B, Tașkiran D, Irkec C, Kutay FZ, Pöğün S. Serum nitric oxide metabolites in patients with multiple sclerosis. J Clin Neurosci 2002; 9: 530-532.

22. Nessler S, Brück W. Advances in multiple sclerosis research in 2009. J Neurol 2010; 257: 1590-1593.

23. Ortiz GG, Macías-Islas MA, Pacheco-Moisés FP, Cruz-Ramos JA, Sustersik S, Barba EA, Aguayo A. Oxidative stress is increased in serum from Mexican patients with relapsing-remitting multiple sclerosis. Dis Markers 2009; 26: 35-39.

24. Rejdak K, Petzold A, Stelmasiak Z, Giovannoni G. Cerebrospinal fluid brain specific proteins in relation to nitric oxide metabolites during relapse of multiple sclerosis. Mult Scler 2008; 14: 59-66.

25. Rogovina NI, Khokhlov AP. Metabolism of lipid peroxidation products in multiple sclerosis patients. Zhurnal Nevropatologii i Psikhiatrii Imeni S. S. Korsakova 1980; 80: 696-700. 
26. Slater TF. Overview of methods used for detecting lipid peroxidation. Methods Enzymol 1984; 105: 283-293.

27. Su KG, Banker G, Bourdette D, Forte M. Axonal degeneration in multiple sclerosis: the mitochondrial hypothesis. Curr Neurol Neurosci Rep 2009; 9: 411-417.

28. Uttara B, Singh AV, Zamboni P, Mahajan RT. Oxidative stress and neurodegenerative diseases: a review of upstream and downstream antioxidant therapeutic options. Curr Neuropharmacol 2009; 7: 65-74.

29. Witherick J, Wilkins A, Scolding N, Kemp K. Mechanisms of oxidative damage in multiple sclerosis and a cell therapy approach to treatment. Autoimmune Dis 2010; 2011: 1-11.

30. Yamashita T, Ando Y, Obayashi K. Changes in nitrite and nitrate levels in cerebrospinal fluid of patients with multiple sclerosis. J Neurol Sci 1997; 153: 32-34.

31. Yuceyar N, Taskiran D, Sagduyu A. Serum and cerebrospinal fluid nitrite and nitrate levels in relapsing-remitting and secondary progressive multiple sclerosis patients. Clin Neurol Neurosurg 2001; 103: 206-211. 